\title{
TTR
}

Traduction, terminologie, rédaction

\section{La traduction intersémiotique dans les dictionnaires du français argotique}

\section{Evangelos Kourdis}

Volume 31, numéro 1, 1er semestre 2018

Traduire la banlieue : problématiques, enjeux, perspectives Translating the Banlieue: Issues, Challenges, Perspectives

URI : https://id.erudit.org/iderudit/1062549ar

DOI : https://doi.org/10.7202/1062549ar

Aller au sommaire du numéro

\section{Éditeur(s)}

Association canadienne de traductologie

ISSN

0835-8443 (imprimé)

1708-2188 (numérique)

Découvrir la revue

Citer cet article

Kourdis, E. (2018). La traduction intersémiotique dans les dictionnaires du français argotique. TTR, 31(1), 127-148. https://doi.org/10.7202/1062549ar
Résumé de l'article

Cet article porte sur l'utilisation de l'illustration en tant que traduction intersémiotique dans les dictionnaires du français argotique. Bien que la présence de l'illustration puisse connoter l'insuffisance de la traduction intralinguale sur laquelle les dictionnaires argotiques du français s'appuient, nous allons voir que l'illustration devient un dispositif de médiation intraculturelle pour le lecteur francophone. Nous examinerons aussi les différents types d'illustration utilisés par les rédacteurs de dictionnaires. Le rôle de l'illustration dans les dictionnaires du français argotique étudiés semble être celui de fournir une orientation sémantique pour le traitement des lexèmes argotiques et leur consolidation dans la mémoire collective culturelle. De même, les diverses formes des illustrations montrent leur valorisation dans la communication intralinguale. 


\title{
La traduction intersémiotique dans les dictionnaires du français argotique
}

\author{
Evangelos Kourdis \\ Université Aristote de Thessalonique
}

\begin{abstract}
Résumé
Cet article porte sur l'utilisation de l'illustration en tant que traduction intersémiotique dans les dictionnaires du français argotique. Bien que la présence de l'illustration puisse connoter l'insuffisance de la traduction intralinguale sur laquelle les dictionnaires argotiques du français s'appuient, nous allons voir que l'illustration devient un dispositif de médiation intraculturelle pour le lecteur francophone. Nous examinerons aussi les différents types d'illustration utilisés par les rédacteurs de dictionnaires. Le rôle de l'illustration dans les dictionnaires du français argotique étudiés semble être celui de fournir une orientation sémantique pour le traitement des lexèmes argotiques et leur consolidation dans la mémoire collective culturelle. De même, les diverses formes des illustrations montrent leur valorisation dans la communication intralinguale.
\end{abstract}

Mots-clés: français argotique, illustration, traduction intersémiotique, dictionnaire

\begin{abstract}
This article deals with the use of illustration as intersemiotic translation in dictionaries of French slang. Even though the presence of illustration may suggest a lack of intralingual translation on which slang dictionaries are built, it shows as we shall see, that illustration becomes an intracultural mediation' mechanism for the French reader. The different types of illustration used by dictionaries' writers are also examined. In the dictionaries of French slang that were analysed, it seems that illustration aims at providing a semantic orientation for the treatment of slang lexemes and their consolidation in the collective cultural memory. Similarly, the diverse forms of illustration show how valorise they are in intralingual communication.
\end{abstract}

Keywords: French slang, illustration, intersemiotic translation, dictionnaire 


\section{Introduction}

Notre étude porte sur l'utilisation de l'illustration ${ }^{1}$ en tant que traduction intersémiotique dans les dictionnaires du français argotique. Pour la réaliser, nous avons examiné les quatre ouvrages suivants: N'ayons pas peur des mots. Dictionnaire du français argotique et populaire de François Caradec (1988), L'argus des mots de Pierre Merle (1997), le Lexik des cités (Azor et al., 2007), «fruit du travail collectif d'un groupe de jeunes originaires d'Évry» (Pérez, 2007, p. 7), et le Petit Dico d'argot de Marc Lemonier (2008)2 Ces dictionnaires présentent des différences qualitatives en ce qui concerne les systèmes sémiotiques non verbaux qu'ils mettent en œuvre. D'une part, ils font appel à divers types d'illustrations : on passe ainsi de dessins purement descriptifs en noir et blanc à des dessins humoristiques (caricatures et bandes dessinées) en couleurs. D'autre part, une amélioration s'observe au fil des décennies dans le graphisme et la typographie, qui attirent l'attention des apprenants du français, surtout des apprenants plus jeunes.

L'inclusion d'illustrations dans les dictionnaires est une pratique établie, mais qui n'est pas largement adoptée. Les dictionnaires demeurent en effet des outils de connaissance essentiellement verbaux. Ceux que nous avons examinés dans le cadre de notre étude sont des dictionnaires intralinguaux; contrairement aux dictionnaires interlinguaux, qui proposent des définitions de mots que les apprenants d'une nouvelle langue connaissent déjà dans leur langue maternelle, les dictionnaires intralinguaux permettent aux locuteurs de découvrir de nouvelles significations dans leur propre langue (Nesi, 1989, p. 126). Le fait que les dictionnaires examinés incluent des illustrations soulève donc d'autant plus de questions.

Il importe, avant d'entrer dans le vif du sujet, d'établir ce que nous entendons par «argot», car ce terme a donné lieu à de multiples définitions. Selon Albert Valdman, «à la suite d'une dérive sémantique, le terme désignera une variété de FP [français populaire] empruntant au vocabulaire du langage des groupes

1. Précisons qu'en sémiotique, on parle plutôt de message iconique. Cependant, nous acceptons la position d'Anne-Marie Drouin selon laquelle «les termes d'“image" et d'“illustration" ne désignent pas des objets différents, mais expriment des regards différents sur un même sujet» (1987, p. 2).

2. Nous tenons à remercier les maisons d'édition Archipel, City et Fleuve Noir, qui nous ont autorisé à reproduire les illustrations contenues dans le présent article. 
sociaux parasitaires exclus de la bonne société» (2000, p. 1181). Il ajoute :

Aujourd'hui, l'argot proprement dit, c'est-à-dire à l'exclusion de ses formes codées, fonctionne comme un réservoir lexical dans lequel puisent les locuteurs de toutes les classes sociales pour rendre leur parler vernaculaire plus expressif.(ibid., p. 1182).

Patrick Charaudeau et Dominique Maingueneau rappellent pour leur part que «la plupart des dictionnaires de langue donnent comme première attestation de ce terme la date de 1628 avec un premier sens de "corporation, confrérie des gueux, des mendiants" " (2002, p. 62). Ils ajoutent que «le terme a connu un élargissement de son acception et qu'on parle désormais d'“argot des jeunes" ou "d'argots de métiers"»(ibid.). Dans le présent article, nous allons utiliser le terme "argot» comme un hyperonyme incluant le vocabulaire argotique, populaire, et ce qu'on appelle de nos jours la langue des jeunes/des cités.

Notre étude vise à répondre à deux principales questions. En premier lieu, quelle est la nécessité de recourir à la traduction intersémiotique dans un dictionnaire en principe intralingual, où la traduction des mots et des expressions argotiques se fait vers le français standard? En second lieu, quel type d'illustrations préfèrent les rédacteurs des quatre dictionnaires examinés pour effectuer cette traduction intersémiotique?

\section{L'intérêt des sémiologues pour les dictionnaires}

L'intérêt des sémiologues pour les dictionnaires n'est pas nouveau. Umberto Eco, notamment, a participé au débat relatif aux deux modèles de représentation du contenu ou de description de la forme de notre connaissance sémiotique, le modèle dictionnaire et le modèle encyclopédie. Eco précise que «l'idéal que s'assigne le dictionnaire est de décrire cette connaissance en termes exclusivement linguistiques, alors que l'encyclopédie entend rendre compte aussi de notre connaissance du monde»(1988, p. 144). Il observe toutefois que les deux modèles tendent à se fondre. À l'instar d'Eco, nous constatons que le message iconique semble être une caractéristique du deuxième type de représentation du contenu, soit le modèle encyclopédie. Eco mentionne que :

Dans la perspective d'une représentation sémantique conforme au modèle encyclopédique, les traits sémantiques, les synonymes, les paraphrases et les instructions contextuelles cessent d'être des 
constructions métalinguistiques pour devenir des interprétants ${ }^{3}$, susceptibles d'être à leur tour interprétés par d'autres interprétants. Est interprétant tout autre signe ou complexe de signes (quelle que soit la substance dans laquelle s'actualise sa forme expressive) qui, dans des circonstances adéquates, traduit le premier signe. (ibid., p. 152)

Ainsi, selon Eco et dans la définition supra, «l'interprétant peut être [...] un signe relevant de sémies et utilisant d'autres substances (un dessin, une couleur)»(ibid.). En parlant de tout autre signe ou complexe de signes indépendamment de leur substance, Eco laisse place à la participation de l'illustration dans le processus de traduction. De même, Jean-Marie Klinkenberg affirme :

La perspective sémiotique impose d'opter pour le modèle encyclopédique: tenter de voir ce qu'il y a de commun entre la manière dont le sens advient à travers toutes les sémiotiques, c'est faire intervenir tous les phénomènes de connaissance. C'est particulièrement le cas dans des sémiotiques non linguistiques. (1996, p. 111)

Comme l'observent Reinhard Hartmann et Gregory James, si la présence d'illustrations est un phénomène «relativement courant» dans les encyclopédies, le même phénomène est «relativement rare» dans les dictionnaires (1998, p. 49; notre trad.). Selon Monika Biesaga, inclure des illustrations dans les encyclopédies et les dictionnaires «est une décision qui comporte un risque parce que leurs rédacteurs révèlent alors ce qui n'appartient pas à leur propre culture» (2017, p. 233; notre trad.).

Les dictionnaires que nous avons étudiés ne sont pas des dictionnaires encyclopédiques. Ils visent plutôt à traduire un écart langagier, ce qui présuppose une connaissance profonde de la norme, de la variété légitime qu'est le français standard, le système sémiotique de référence vers lequel l'écart est traduit. L'inclusion d'illustrations pourrait-elle ainsi poser l'insuffisance de la norme?

3. Eco utilise une notion proposée par Charles Sanders Peirce. Selon Peirce, «un signe, ou representamen, est quelque chose qui tient lieu pour quelqu'un de quelque chose sous quelque rapport et a quelque titre. Il s'adresse à quelqu'un, c'est-à-dire crée dans l'esprit de cette personne un signe équivalent ou peut-être un signe plus développé. Ce signe qu'il crée, je l'appelle interprétant du premier signe. Ce signe tient lieu de quelque chose : de son objet. Il tient lieu de cet objet, non sous tous rapports, mais par référence à une sorte d'idée que j'ai appelée quelquefois le fondement du representamen»(Peirce, 1979, p. 121). 
La langue française présentée serait-elle une réalité inconnue? Nous tâcherons de répondre à ces questions dans notre analyse.

\section{La notion de traduction intersémiotique}

Les termes «traduction intersémiotique» et "transmutation» ont été introduits dans le champ des études linguistiques, sémiotiques et traductologiques par Roman Jakobson. Jakobson définit la traduction intersémiotique ou transmutation comme "l'interprétation des signes linguistiques au moyen de systèmes de signes non linguistiques» $\left(1963\right.$, p. 79) ${ }^{4}$. Si l'on considère que, pour Jakobson, «la traduction implique deux messages équivalents dans deux codes différents» (ibid., p. 80), il en résulte que le message transmis par l'illustration doit être équivalent au message porté par le système linguistique. Ceci semble difficile si, à l'instar d'Eco, on tient compte du fait qu' «un système sémiotique donné peut dire moins ou plus qu'un autre système sémiotique, mais on ne peut affirmer que tous deux peuvent exprimer les mêmes choses» (2007, p. 381). Bien sûr, cette constatation n'exclut pas le caractère médiateur de l'illustration dans le dictionnaire.

Il est généralement admis que, dans le passage d'un système sémiotique à un autre, il y a une perte de la charge informationnelle, comme cela se produit dans la traduction. La sémiotique considère la traduction comme un acte entièrement sémiotique. Pour certains sémiologues dont Greimas, on recourt à la traduction intersémiotique à cause de la complexité du message linguistique. Greimas affirme que «tout ensemble signifiant de nature différente de celle de la langue naturelle peut être traduit, avec plus ou moins d'exactitude, dans une langue naturelle quelconque: ainsi la peinture et sa traduction par la critique picturale» (1966, p. 12). I1 soutient en outre que «les langues naturelles [...] servent de point de départ à des transpositions et de point d'aboutissement à des traductions», soulignant "la complexité de l'ensemble signifiant qu'est une langue naturelle» (ibid., p. 13). L'argot étant un système linguistique complexe composé de plusieurs registres, langages codés, mots étrangers ou francisés, le recours à l'illustration en tant

4. Klinkenberg, en élargissant la notion de fonction métalinguistique du message (exprimée pour la première fois par Jakobson en 1963), parle de «fonction metasémiotique»; il nomme ce phénomène d'interprétation «metasémiotique hétérosémiotique». En vertu de celui-ci, «un message performé à partir du code $a$ parle d'un autre code $b$ (les partitions renvoient à la musique, les mots à la peinture)» (1996, p. 57). 
que traduction intersémiotique semble justifié. La traduction intersémiotique est incluse dans ce que Paolo Fabbri définit comme des «articulations de sens à travers les différentes substances d'expression» (2008, p. 29), une position qui s'identifie entièrement à celle d'Eco mentionnée plus haut.

Toutes ces positions amènent à l'utilisation d'illustrations dans les dictionnaires du français argotique, une pratique qui ne semble pas très répandue dans les dictionnaires européens, mais qui s'avère fréquente dans les dictionnaires américains (Rey-Debove, 1971, p. 34; Nesi, 1989, p. 126).

\section{Les fonctions de l'illustration}

Un certain nombre de sémiologues (Barthes, 1964; Eco, 2003; Fabbri, 2008; Petrilli et Ponzio, 2012; Kourdis et Kukkonen, 2015, etc.) et de traductologues (Baker, 1992; Munday, 2004; Pereira, 2008; Guidère, 2008; Gambier, 2016, etc.) semblent considérer que l'illustration a des effets positifs lorsqu'elle est utilisée comme support dans le processus de traduction ${ }^{5}$. C'est Roland Barthes qui, le premier, a étudié l'image d'un point de vue sémiologique dans le cadre des relations entre messages linguistiques et iconiques. Il écrit à ce sujet :

La liaison du texte et de l'image est fréquente; cette liaison semble avoir été peu étudiée d'un point de vue structural; quelle est la structure signifiante de l'illustration"? L'image double-t-elle certaines informations du texte, par un phénomène de redondance, ou le texte ajoute-t-il une information inédite à l'image? (Barthes, 1964, p. 43). ${ }^{6}$

Cette redondance de l'illustration par rapport au texte peut vraisemblablement faciliter la démarche cognitive des utilisateurs de dictionnaires, en plus d'exercer une fonction positive sur le plan psychologique. Jean-René Ladmiral fait remarquer que «l'image n'est pas seulement une sorte de "signifié-pivot" contribuant à la circulation de l'information, elle a aussi une fonction psychologique» (1994, p. 30). L'aspect cognitif de l'illustration est également souligné par Anne Marie Drouin :

5. Certains théoriciens de la traduction ont cependant des réserves sur ce type de traduction que Jakobson a nommé «traduction intersémiotique».

6. Selon Martine Joly, «la complémentarité des images et des mots réside aussi dans le fait qu'ils se nourrissent les uns les autres. Nul besoin d'une coprésence de l'image et du texte pour que ce phénomène existe. Les images engendrent des mots qui engendrent des images dans un mouvement sans fin» (1993, p. 106). 
dans un texte de vulgarisation ou dans un manuel, les images jouent souvent le rôle d'illustrations, ces illustrations ayant comme but d'expliquer, d'aider à la mémorisation, et d' «accrocher» le regard, éventuellement à travers un jeu sur l'esthétique [...]. (1987, p. 2)

Est-ce cette fonction psychologique de l'image qui fait qu'elle est utilisée pour accompagner des traductions intralinguales dans les dictionnaires du français argotique, ou doit-on classer le dictionnaire du français argotique parmi les textes de vulgarisation?

L'approche de l'illustration comme traduction intersémiotique n'est pas récente. Envisageant les illustrations littéraires sous l'angle de la traductologie, Nilce Pereira laisse entendre que «les figures des livres illustrés sont des traductions (intersémiotiques) du texte et qu'elles peuvent être analysées à l'aide des outils qui sont appliqués à la traduction verbale» (2008, p. 104; notre trad.). Elle décrit par ailleurs trois façons précises dont les illustrations peuvent traduire le texte : en reproduisant littéralement les éléments textuels sur la figure, en mettant en relief un élément spécifique du récit et en adaptant les figures à une idéologie donnée ou à un courant artistique. Riitta Oittinen relève aussi de nombreuses similarités entre la traduction (dans le verbal) et l'illustration (traduction dans l'image) en tant que formes d'interprétation (2000, p. 106). On pourrait justifier l'illustration dans la littérature comme une détente psychologique dans un texte en principe verbal. Mais c'est dans le texte verbal que réside l'intérêt des traductologues, qui considèrent la traduction comme une opération et un produit avant tout verbal. Pour Mona Baker, l'utilisation de l'illustration comme stratégie de traduction s'impose dans certains cas précis. Il s'agit selon elle d'«une option utile si le mot qui n'a pas d'équivalent dans la langue cible renvoie à une entité physique qui peut être illustrée, surtout s'il existe des restrictions sur l'espace et si le texte doit rester court, concis et au point»(Baker, 1992, p. 42; notre trad.). Ali Almanna estime pour sa part que l'emploi de l'illustration comme stratégie de traduction est lié à la fonction d'élucidation que remplit l'illustration, fonction décrite par Barthes en 1964. Almanna commente cette fonction comme suit :

les traducteurs recourent à la traduction par l'illustration pour des raisons différentes: pour éviter une confusion, pour refléter une dimension sémiotique, pour répondre aux exigences de la langue cible pour une meilleure compréhension, etc. (2016, p. 77; notre trad.) 
En lexicographie, la fonction de redondance de l'illustration est évoquée par Josette Rey-Debove, qui explique:

la fonction de l'illustration est surtout définitionnelle [...]. Si la définition linguistique est suffisante, l'illustration aide à l'évocation du concept, c'est une information d'appoint. La définition est analytique, l'illustration plutôt synthétique. (1971, p. 35).

Enfin, pour Robert Ilson, l'illustration fait partie des cinq techniques explicatives de base utilisées dans les dictionnaires, les quatre autres étant l'exemplification, la discussion, la définition et la traduction (1987, p. 193).

Ainsi, lorsqu'il est question de l'illustration, on parle d'une technique explicative ou d'un choix qui dépasse les restrictions imposées par le texte, ou l'on évoque sa fonction de redondance, sa fonction définitionnelle ou sa fonction d'élucidation. Dans les dictionnaires que nous avons examinés, l'illustration fonctionne comme une traduction intersémiotique qui aide l'utilisateur à saisir le sens des mots et expressions argotiques, en interrompant la monotonie du système sémiotique de la langue, ajoutant un ton différent dans un processus de traduction qui exige des manipulations linguistiques particulières.

\section{Les différents types d'illustrations dans les dictionnaires du français argotique}

Dans les dictionnaires que nous avons examinés, les illustrations correspondent à des dessins en noir et blanc ou en couleurs. Ce choix n'est pas un hasard. En effet, comme l'observe Drouin :

Le dessin est plus général et plus proche du concept que la photo, même s'il reste fidèle à la réalité, dans la mesure où, encore plus que la photo, il est le résultat d'un choix parmi les éléments jugés significatifs, il est une simplification et une mise en évidence de ce qui est essentiel. (1987, p. 5)

Jean Baptiste Bon Boutard définit le dessin comme une «image tracée au crayon ou à la plume» (1826, p. 217). Il ajoute qu'on appelle «dessin au trait» un dessin "qui ne figure que la délinéation des objets sans exprimer leur relief par le clair-obscur» (ibid.). Klinkenberg précise à propos du dessin au trait que «le style "ligne claire" en bande dessinée [en] donne une bonne idée. Entre les traits, c'est en fait le vide : toute l'information est concentrée dans les zones délimitant les plages» (1996, p. 399). 
Les caractéristiques propres au dessin connotent la subjectivité de l'auteur du dictionnaire, qui a la possibilité de choisir le type de dessin qui pourra le mieux représenter le sens du lexème traité. Michel Proces note que, comparativement aux photos ou au dessin informatisé, «le dessin au trait [...] reste le plus lisible et [celui] qui supporte le mieux les outrages du temps» (1997, p. 173). Proces précise en outre que «le dessin au trait présente également d'indéniables facilités de reproduction, ce qui constitue un avantage pour la diffusion des connaissances» (ibid.).

Des quatre dictionnaires étudiés, un seul contient des dessins en couleurs. Nous savons que les dessins en noir et blanc réduisent les coûts d'impression; de plus, comme le remarque Robert Lew, «il n'y a pas de preuve que les illustrations colorées dans les dictionnaires sont plus efficaces que les dessins traditionnels» (2010, p. 299; notre trad.).

Nous proposons la typologie suivante, établie selon le style des dessins présents dans les dictionnaires examinés, pour classer les illustrations : les illustrations de type scientifique, les illustrations de type culturel, les illustrations de type caricatural, les illustrations de type artistique et les illustrations de type bande dessinée.

\subsection{Les illustrations de type scientifique}

Cette première catégorie est représentée dans N'ayons pas peur des mots. Dictionnaire du français argotique et populaire de François Caradec (1988). Les illustrations qu'on y trouve sont de Denis Horvath Morez et elles sont peu nombreuses. Elles figurent le plus souvent en bas de page, sous forme de petits dessins humoristiques accompagnant le texte et ayant une fonction de redondance. Cependant, les illustrations de type scientifique ne correspondent pas à ce modèle : elles consistent en des dessins en noir et blanc qui occupent toute la page et qui semblent privés de l'aspect humoristique. C'est en raison des sujets qu'elles abordent que nous les qualifions de scientifiques. Comme le mentionnent Phyllis Wood et Patrick McDonnell :

Lillustration scientifique est produite pour un type spécifique de communication visuelle dans la science. [...] L'artiste doit bien connaître le sujet et dessiner de manière sélective et interprétative, sans enregistrer tout photographiquement [...]. Bien que l'illustration scientifique soit sérieuse et factuelle, elle n'a pas besoin d'être manipulée dans un style sec ou monotone. Elle peut et doit être 
conçue de manière aussi réfléchie et innovante que n'importe quelle œuvre d'art, en utilisant les styles et les méthodes actuels, et en combinant des techniques traditionnelles et informatiques. (Wood et McDonnell 1994, p. vii; notre trad.)

Les illustrations de type scientifique contenues dans le dictionnaire de Caradec concernent, par exemple, l'anatomie de l'homme (Caradec, 1988, p. 55) et de la femme (ibid., p. 56); elles s'apparentent alors aux planches de manuels d'anatomie. Des parties précises du corps sont identifiées par un numéro suivi d'un mot, le numéro pointant vers la partie du corps en question. Par exemple, dans l'illustration relative à l'anatomie de l'homme, le numéro 2 est suivi du mot "pif» et pointe vers le nez, le numéro 17 est suivi du mot «bide» et pointe vers l'abdomen, et ainsi de suite. Ce qui est intéressant, c'est que l'auteur ne se limite pas à traduire intersémiotiquement les parties du corps (par exemple, «les roustons ", numéro 20, par l'indication des parties correspondantes dans le dessin) : il donne aussi des synonymes («les agobilles», «les balloches», les «bonbons», etc.), essayant de couvrir toute la gamme de termes argotiques français qui correspondent aux parties en question.

Cette intersémioticité s'inscrit dans la fonction informative ou référentielle de l'illustration. Martine Joly explique à ce propos :

La fonction informative (ou référentielle), souvent dominante dans l'image, peut aussi s'amplifier en une fonction épistémique, lui donnant alors la dimension d'outil de connaissance. Outil de connaissance, parce quelle donne bien sûr des informations sur les objets, les lieux ou les personnes, sous des formes visuelles aussi diverses que les illustrations, les photographies, les plans ou encore les panneaux. (1993, p. 50; en italique dans l'original)

On doit toutefois admettre que l'intersemioticité dans le cas de l'illustration des sèmes "anatomie de l'homme» et "anatomie de la femme» du dictionnaire de Caradec balance entre la scientificité et la vulgarisation. D'une part, l'illustration évoque visuellement des manuels de médecine, d'anthropologie ou de biologie (le sème «anatomie» évoque aussi ces sciences); d'autre part, elle constitue une façon de communiquer des lexèmes vulgaires et permet d'éviter leur traduction intralinguale en français standard ${ }^{7}$. De plus, malgré

7. Comme l'observe Lew, «tout le contenu du vocabulaire ne peut pas toujours être utile ou illustré dans un dictionnaire» (2010, p. 297; notre trad.). 
le fait que le dictionnaire fournit un grand nombre de synonymes, seul un lexème est mentionné dans l'illustration, probablement le plus courant, ce qui constitue l'archilexème selon le rédacteur du dictionnaire.

Par ailleurs, des images semblables à celles qui sont utilisées par Caradec pour illustrer l'anatomie de l'homme et de la femme figurent souvent dans les manuels d'enseignement et d'apprentissage de langues étrangères pour décrire les parties du corps humain. Dans ces cas, la figure est par contre soit habillée, soit présentée d'une manière plus générale si elle est nue, ce qui permet d'éviter de nommer les parties du corps caractérisées comme étant "privées» ou «sensibles». Le fait que cette réserve ne soit pas reproduite dans le dictionnaire de Caradec révèle que l'argot, dépassant les tabous culturels, est un système linguistique riche, avec des mots qui pourraient être perçus ailleurs comme «choquants », voire inconnus pour un certain nombre de lecteurs.

\subsection{Les illustrations de type culturel}

Selon Annette Klosa, «l'information culturelle [...] contenue dans un dictionnaire peut apparaître dans la définition, mais aussi dans les exemples et les collocations du lemme» (2015, n.p.; notre trad.). On trouve des illustrations de type culturel dans le dictionnaire de Caradec (1988) ainsi que dans le Lexik des cités (Azor et al., 2007). Celles-ci représentent notamment des gestes. La gestuelle, on le sait, dépend de codes culturels et a donné lieu à des recherches sémiotiques concernant le langage muet, les diverses positions du corps, les gestes rituels, la pantomime, etc. (Eco, 1984). Dans le dictionnaire de Caradec, une illustration concerne spécifiquement le sème "gestes» $(1988$, p. 93). Elle comprend un ensemble de six dessins représentant un homme qui réalise des gestes précis; la signification de ces gestes (dans la culture française) est indiquée en légende, sous chaque dessin, ce qui constitue une traduction intersémiotique de ces gestes. Par exemple, l'un des dessins correspond à l'expression «chut!»: l'homme y est représenté avec l'index de la main droite levé et placé sur la bouche. Dans le Lexik des cités (illustration 1, page suivante), nous voyons que pour expliquer le lexème "tchek», qui est défini comme un "geste de complicité», un "geste de salutation» ou un "geste qui conclut un accord» (ibid., p. 325), les rédacteurs présentent des illustrations colorées qui décrivent ces gestes sans utiliser de légende. 


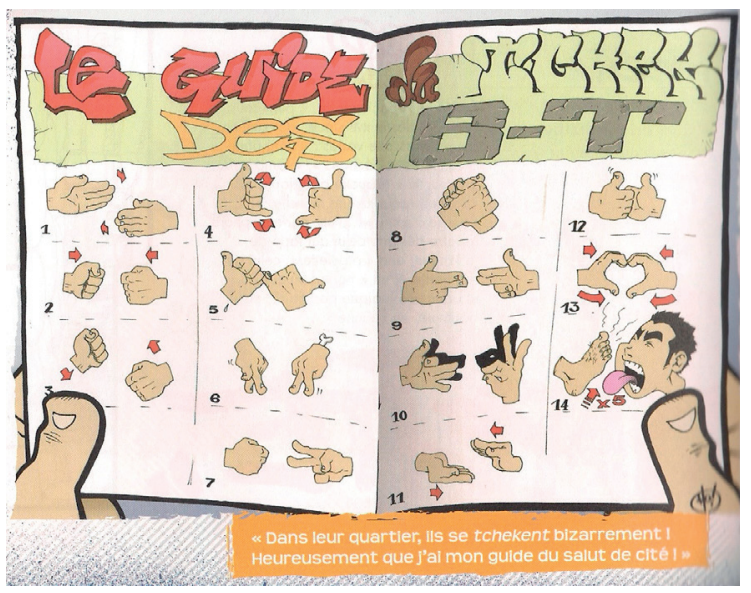

Illustration $1:$ le sème «tchek"

(C) Azor et al., Lexik des cités, Fleuve Noir, 2007, p. 324

Il est évident que les auteurs du Lexik des cités accordent de l'importance à la synergie des systèmes sémiotiques du dessin et de la couleur. Il est d'ailleurs mentionné dans l'ouvrage que les dessins sont de Cédric Nagau et leur colorisation, d'Alhassane Sarré et Alhousseynou Sarré. Malgré l'intersémioticité produite entre les dessins et les définitions, l'absence de légende crée une certaine confusion dans leur correspondance. Le commentaire qui figure sous l'illustration - «Dans leur quartier, ils se tchekent bizarrement! Heureusement que j'ai mon guide du salut de cité» - indique qu'il s'agit d'un guide du salut, mais n'explique pas quel dessin correspond à quel geste. Et cela, même si les dessins sont très descriptifs et utilisent parfois d'autres systèmes sémiotiques non verbaux comme les flèches rouges pour mieux décrire les gestes.

La gestualité est un système sémiotique profondément culturel et difficile à décrire. Greimas en distingue deux types : la gestualité de communication directe et la gestualité de transposition. Le second type ne pouvant être exploité en vue de la communication que grâce aux procédures de transposition de signifiants, il se subdivise en gestualité mimétique et gestualité ludique, selon les dimensions des unités transposables - signes ou énoncés (Greimas, 1968, p. 28). Les dessins qui représentent des gestes dans le dictionnaire de Caradec (1988, p. 93) pourraient être classés dans le premier 
type, car les gestes qu'ils représentent sont très descriptifs. Par contre, les dessins provenant du Lexik des cités présentent un aspect ludique (surtout les dessins $\mathrm{n}^{\text {os }} 6,10,13$ et 14 dans l'illustration 1 ci-contre) et correspondent donc à une gestualité de transposition. La traduction intersémiotique de la gestualité peut ainsi être illustrée de différentes façons dans les dictionnaires argotiques.

La figure incluse dans le dictionnaire de Caradec pour représenter le sème «gestes» $(1988$, p. 93) correspond à un homme adulte qui n'a rien de particulier; elle connote l'aspect représentatif de la gestualité. Dans le Lexik des cités, la gestualité est adoptée par un groupe social plus limité, celui des cités. Une telle différentiation dans l'illustration de la gestualité montre la complexité de la communication gestuelle, même dans un milieu culturel commun (français et argotique).

\subsection{Les illustrations de type caricatural}

Les illustrations de cette catégorie correspondent à des dessins humoristiques qui se concentrent intersémiotiquement sur un mot argotique. Selon Salvatore Attardo, une condition préalable à la production de l'humour est l'existence d'un code sémiotique, puisque «l'humour existe seulement dans la communication qui est la sémiose» (2001, p. 32; notre trad.). Cela veut dire que les choses ne sont pas en elles-mêmes humoristiques : elles doivent être codifiées et perçues comme signes d'un code. L'intertextualité et la narration semblent être deux procédés qui contribuent à cette tâche.

Le premier exemple que nous proposons provient du dictionnaire de Pierre Merle, L'argus des mots (1997). Notons que l'auteur ne fait aucune allusion aux illustrations incluses dans son dictionnaire, bien que le nom de leur créateur, Bruno Dollone, soit mentionné sur la couverture. Dans l'illustration 2 (page suivante), une jeune fille se couche près d'un loup. L'intertexte auquel se réfère le dessin est le conte de tradition orale Le Petit Chaperon rouge, dont la première version écrite est de Charles Perrault. Dans le phylactère du dessin apparaît le mot «burnes ", qui appartient au sème des «testicules", transformant les paroles du fameux conte en un énoncé du type palimpseste verbo-culturel. L'effet humoristique est aussi évident dans l'énoncé hors phylactère, qui peut être catégorisé dans le discours implicite. 


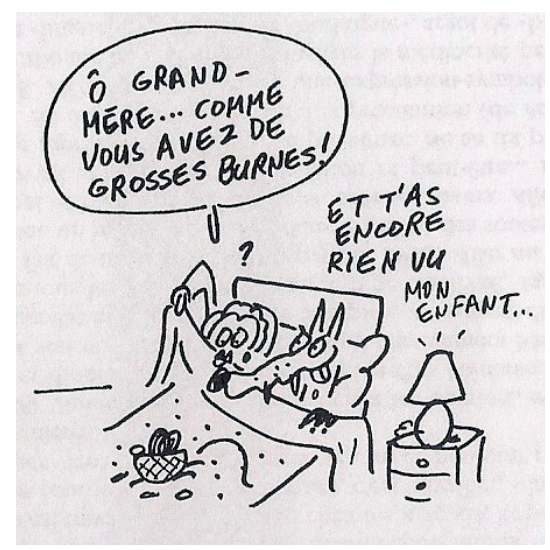

Illustration 2 : le sème «testicules»

(C) Bruno Dolonne - Pierre Merle. L'argus des mots, L'Archipel, 1997, p. 258

Alors que Caradec a choisi le lexème «roustons» pour représenter le sème «testicules» dans le dessin scientifique inclus dans son dictionnaire $(1988$, p. 55), la préférence de Merle va ici au lexème «burnes». C'est donc dire qu'il y a deux traductions intersémiotiques différentes pour le même sème, qui compte d'ailleurs plus de cinquante synonymes classés en sept catégories (hors cote, désuet, kitsch, pittoresque, classique, météorique et néoprécieux); «burnes» figure dans la catégorie "classique». Cette classification montre la richesse du vocabulaire argotique et le fait que, parfois, même par le dessin, les auteurs de dictionnaires évitent d'illustrer des lexèmes qui proviennent d'une catégorie vulgaire qui pourrait être choquante pour le lecteur.

Nous observons ce phénomène dans l'illustration 3, tirée du Lexik des cités et qui se réfère au sème "postérieur de la femme». Dans ce cas précis, les auteurs expliquent le lexème «boule» à l'aide du lexème "postérieur» et ils donnent pour synonyme «seufs» ${ }^{8}$. La légende du dessin décrit la situation de communication dans laquelle le mot est utilisé : «L'autre soir, j’ai croisé une meuf, elle m'a souri. Tu me connais : j'ai regardé son boule et j'ai fait demitour...» Le ton humoristique est présent dans ce dessin. Comme

8. Dans le dictionnaire de Caradec (1988, p. 56), c'est le lexème «cul» qui est inclus dans l'illustration pour les postérieurs de femme, un lexème qui, comparé à «boule», est peut-être considéré plus reconnaissable et vulgaire. 
l'observe Lilian-Atieno Gangla (2001), il n'y a pas d'accord entre les lexicographes sur la question de savoir si la couleur ajoute du réalisme, contribuant ainsi au processus de compréhension du sens, ou si elle distrait l'attention du lecteur. D'après nous, la couleur ajoute dans ce cas un ton léger à l'illustration.

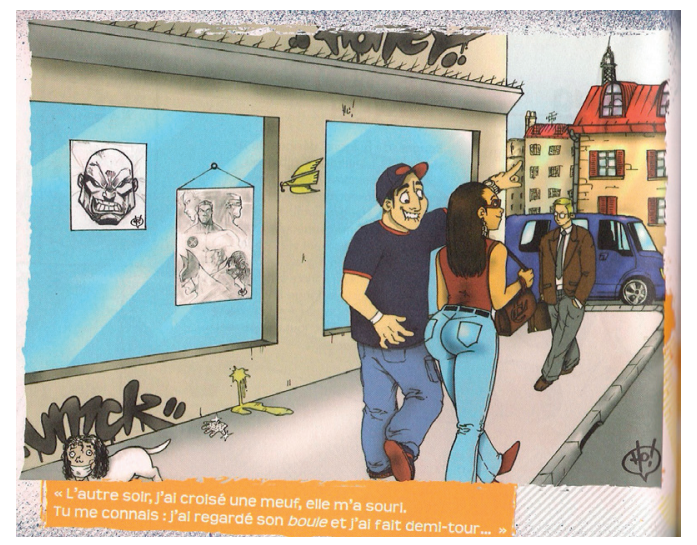

Illustration 3 : le sème "postérieur de la femme"

(C) Azor et al., Lexik des cités, Fleuve Noir, 2007, p. 78

Dans l'illustration 4 ci-dessous, un couple essaie de répondre à une question concernant le sème "anus", probablement tirée d'un jeu de mots croisés.

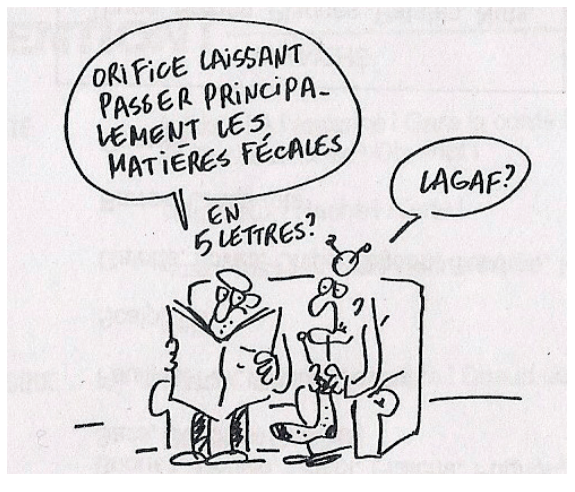

Illustration $4:$ le sème «anus "

(C) Bruno Dolonne - Pierre Merle. L'argus des mots, L'Archipel, 1997, p. 23 
La réponse de la femme - «lagaf» - est d'une charge culturelle élevée : le lecteur doit bien connaître la réalité culturelle française de l'époque pour décoder cette réponse et en saisir la portée humoristique. En effet, «lagaf» n'est pas un lexème synonyme d'anus; c'est plutôt le nom d'un humoriste et animateur de télévision, Vincent Lagaf. Le fait que l'illustration ne contient pas de synonyme tenant lieu d'explication et qu'elle dépasse les limites strictement linguistiques d'un dictionnaire en offrant une réponse subjective basée sur la culture quotidienne renforce l'aspect humoristique, mais ne contribue pas à la compréhension et à la connaissance linguistique du lecteur.

De même, le dessin humoristique qui accompagne le sème "vêtements» dans le dictionnaire de Caradec (1988, p. 61) ne contribue pas à l'enrichissement des connaissances linguistiques du lecteur, puisqu'il présente un homme accroché à une corde à linge avec des vêtements mis à sécher. Les vêtements dans cette image constituent une traduction intersémiotique du sème "vêtements", mais le dessin n'illustre aucun mot du français argotique. On peut penser que ces deux illustrations - pour le sème "anus" (Merle, 1997, p. 23) et le sème "vêtements» (Caradec, 1988, p. 61) -, la première sans traduction véritable, la deuxième étant une traduction intersémiotique d'un lexème ordinaire et non argotique, se caractérisent par une fonction décorative. En renforçant l'aspect humoristique, elles contribuent à la détente du lecteur et suggèrent, peut-être, que la lecture d'un dictionnaire argotique est une tâche difficile.

\subsection{Les illustrations de type artistique}

Les illustrations de type artistique sont les moins fréquentes dans les dictionnaires que nous avons examinés. En fait, on en trouve seulement dans le Petit Dico d'argot de Marc Lemonier (2008), et le dessinateur n'est pas nommé. Dans ces cas, le dessin en noir et blanc accompagne un texte de type encyclopédique. Ainsi, dans l'illustration 5 reproduite ci-dessous, le lexème «zapper» est défini par la présentation d'une situation de communication dans laquelle il est utilisé. Or, l'illustration constitue la traduction intersémiotique du premier sens du lexème, qui s'applique à la télévision, et non celle du sens argotique mentionné dans le texte entre guillemets, à savoir «je n'ai pas fait attention à ce que tu as dit ou fait, car j'avais l'esprit ailleurs». 


\section{ZAPPER}

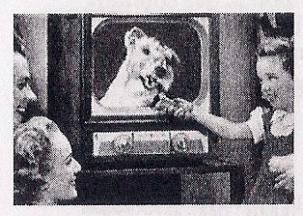

Verbe. Changer de sujet ou de situation.

La télévision elle aussi a imposé son vocabulaire. En zappant pour changer de chaîne, on dispose d'un pouvoir que l'on souhaiterait avoir dans la vie : pouvoir changer de travail, de partenaire sexuel, de ville... Malheureusement, ce n'est pas le cas, aussi zapper signifie surtout « je n'ai pas fait attention à ce que tu as dit ou fait, car j'avais l'esprit ailleurs ».

Illustration $5:$ le sème «zapper»

(C) Marc Lemonier, Petit Dico d'argot, City, 2008, p. 208

Dans l'illustration 5, un dessin de type artistique représente une fillette avec ses parents devant la télévision et rappelle une scène familiale des années 1950. Pourtant, dans les années 1950, il n'y avait pas de télécommande pour zapper. L'art offre cette perspective de liberté expressive, et cette liberté ne semble pas empêcher le lecteur d'associer le lexème "zapper» à l'illustration.

De même, Lemonier présente analytiquement une situation de communication pour décrire le lexème «cravater» (illustration 6), en plus de fournir des synonymes.

\section{Cravater}

Verbe. Arrêter.

Le vocabulaire de l'arrestation se construit souvent à partir de la partie du corps ou de ses vêtements par laquelle on est aggrave : alpaguer par le bras ou le costume, cravater par le cou, symbolisé ici par le port de la cravate. Notons, ce qui n'a rien à voir, qu'une « cravate de notaire» est une pratique sexuelle parfois dénommée aussi « Napoléon sur les remparts ".

SYNONYMES : ALPAGUER ; POISSER; SERRER.

Illustration 6 : le sème «cravater»

(C) Marc Lemonier, Petit Dico d'argot, City, 2008, p. 73

Le dessin représente l'arrestation d'une personne par la police de l'époque (peut-être le $\mathrm{XIX}^{\mathrm{e}}$ siècle). La traduction intersémiotique ne se limite pas au lexème "cravater»; elle s'étend à des synonymes comme "alpaguer» et inclut ainsi des informations culturelles supplémentaires. Nadine Celotti observe que, dans les dictionnaires, «de nombreuses informations comme des données narratives sont ajoutées pour mieux illustrer la charge culturelle intrinsèque de 
certains mots» $(2008$, p. 218) 9 . Enfin, comme dans le cas précédent, l'illustration 6 localise intersémiotiquement l'événement dans une époque non contemporaine, peut-être pour connoter l'ancienneté de certains termes argotiques.

\subsection{Les illustrations de type bande dessinée}

Au niveau iconique, l'illustration de type bande dessinée apparaît seulement dans le Lexik des cités. Dans l'avant-propos de cet ouvrage, Marcela Pérez souligne que «ce qui a particulièrement motivé le groupe d'auteurs est le risque de malentendus» (2007, p. 8). Les illustrations de ce genre impliquent la mise en situation des lexèmes afin d'en illustrer le sens, ce qui permet de révéler d'une part leur sens connu ou figé et, d'autre part, leur sens argotique. Comme mentionné par Pérez, «c'est le fossé générationnel qui a inspiré le comic-strip récurrent mettant en scène une mère et sa fille dans un dialogue impossible» (ibid.; italique dans l'original). D'ailleurs Pérez ajoute que le choix des personnages «n'est pas innocent» (ibid.) : il vise à ce que le lecteur s'attache à cette mère «qui fait des efforts en s'essayant au verlan, et à cette adolescente qui laisse entrer sa mère dans son univers» (ibid.).

Dans l'illustration 7 ci-contre concernant le lexème «limer», on observe que la bande dessinée est composée de deux cases : la première case présente le lexème employé au sens argotique, qui signifie «faire l'amour» (synonyme «herrek»), un sens que seule l'adolescente connaît (Azor et al., 2007, p. 218). La deuxième case présente la mère qui écoute sa fille employer le lexème «limer» et qui l'associe à la lime à ongles utilisée pour faire une manucure. On comprend que les deux univers, adulte et adolescent, sont à part. Les rédacteurs du dictionnaire soulignent que «si au moins le doute sur le véritable sens des mots s'installait dans l'esprit du lecteur, c'est sûr, les efforts d'imagination sur ces dialogues frustrés auront valu la peine» (Azor et al., 2007, p. 8).

La binarité est bien exploitée dans le Lexik des cités. Les deux univers, celui de la mère qui représente la génération adulte (le passé) et celui de sa fille qui représente la génération adolescente (le nouveau), sont présentés ensemble mais restent distincts. Peutêtre que la fille peut comprendre le sens figé et le sens argotique,

9. Cette stratégie est évidente également dans le Lexik des cités. Margarito affirme qu' «entre la lexicographie science savante et les productions de la linguistique populaire, [il y a une] zone pour laquelle nous venons d'avancer l'appellation de para-lexicographie» (2007, p. 172). 
mais sa mère, de la génération précédente, comprend seulement le sens figé. Il est à noter que ce dictionnaire est né «en tant que projet "pédagogique" collectif pour donner directement la parole à ces jeunes et faire (re)connaître leur identité à travers les mots » (Celotti, 2008, p. 210).

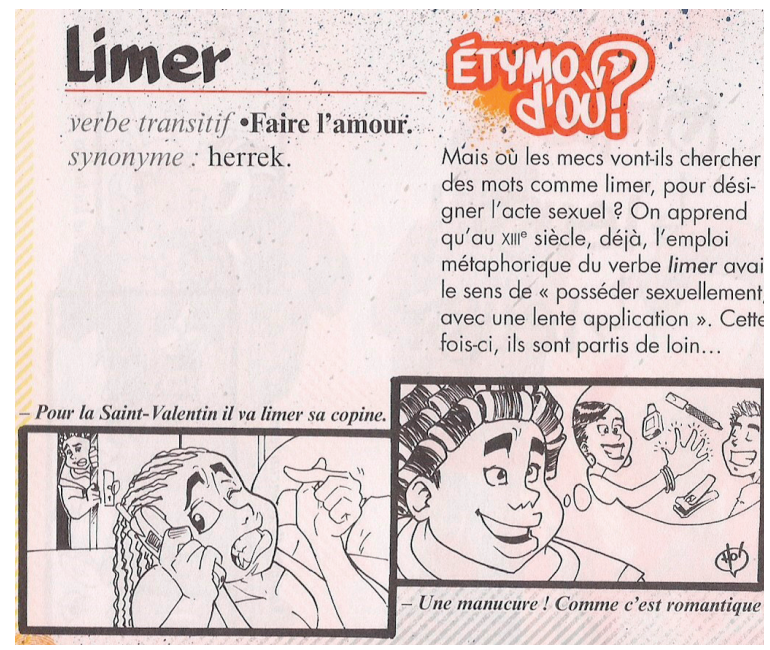

Illustration 7 : le sème "limer»

(C) Azor et al., Lexik des cités, Fleuve Noir, 2007, p. 218

\section{Conclusion}

Par sa nature polysémiotique, l'illustration est un système sémiotique différent de celui de la langue. Quand un mot argotique passe d'un système sémiotique à l'autre, du linguistique à l'iconique, cela peut connoter sa consolidation dans la mémoire culturelle. Ainsi, nous pourrions conclure que «les roustons », «les burnes» ou «les boules» sont des mots plus fréquents ou plus connus comparativement aux mots «agobilles», «balloches", "pralines», etc. L'illustration peut devenir un moyen de diffusion du vocabulaire argotique, mais cette diffusion est sélective, puisqu'elle laisse peu de place au système sémiotique de la langue ayant besoin d'un archilexème. Qui plus est, l'illustration pose des limites à la vulgarisation de certains lexèmes argotiques. Il est peut-être facile de prononcer des mots ou des expressions argotiques, mais il est difficile de les visualiser, et c'est pour cela que les auteurs des dictionnaires semblent faire preuve de réserve quant au choix des lexèmes qui sont inclus dans les dessins ou qui les accompagnent. Il est aussi intéressant de voir que 
l'illustration ne traduit pas toujours intersémiotiquement le lexème argotique. Assez souvent, elle traduit des synonymes qui peuvent aussi appartenir au vocabulaire argotique. Nous pourrions ainsi affirmer que l'illustration oriente le sens. Il semble que la traduction intersémiotique aide le lecteur du dictionnaire à dépasser certains obstacles de compréhension concernant le vocabulaire argotique ou d'équivalence avec la norme.

Dans les dictionnaires que nous avons examinés, le rôle de l'illustration tient à l'orientation sémantique entre les lexèmes argotiques et à leur consolidation dans la mémoire culturelle collective. La variété de formes des illustrations montre en outre leur valorisation dans la communication intralinguale. Dans la fonction d'ancrage telle que décrite par Barthes en 1964, le texte dirige le lecteur entre les signifiés de l'image, et le lecteur peut ainsi faire une sélection parmi les signifiés. Nous croyons que l'illustration dans les dictionnaires du français argotique fonctionne de manière inverse : elle dirige le lecteur vers les lexèmes argotiques en essayant d'établir un archilexème. Ces constatations tendent à montrer que l'illustration est appelée à jouer un rôle important dans les dictionnaires monolingues spécialisés et qu'elle continuera de susciter l'intérêt des chercheurs.

\section{Références}

Almanna, Ali (2016). The Routledge Course in Translation Annotation: Arabic-English-Arabic. Londres et New York, Routledge.

Attardo, Salvatore (2001). Humorous Texts: A Semantic and Pragmatic Analysis. Berlin, Mouton de Gruyter.

Azor, Cindy, Marie Mirline Azor, Franck Longepied, Cédric Nagau, Marcela Pérez, Imane Rajef, Alhassane Sarré, Alhousseynou Sarré, Boudia Sylla, Kandé Sylla et Dalla Touré (2007). Lexik des cités. Précédé d'un dialogue entre Alain Rey et Disiz la Peste. Paris, Éditions Fleuve Noir.

Baker, Mona (1992). In Other Words. A Coursebook on Translation. Londres et New York, Routledge.

Barthes, Roland (1964). «La rhétorique de l'image». Communications, 4, p. 40-51.

Biesaga, Monika (2017). «Pictorial Illustrations in Encyclopaedias and in Dictionaries: a Comparison». In I. Kosem et al., dir. Electronic Lexicography in the 21st Century. Proceedings of eLex2017 Conference. Lexicography from Scratch. Brno, Elex, p. 221-236.

Boutard, Jean-Baptiste-Bon (1826). Dictionnaire des arts du dessin: la peinture, la sculpture, la gravure, et l'architecture. Paris, Le Normant. 
Caradec, François (1988). N'ayons pas peur des mots. Dictionnaire du français argotique et populaire. Paris, Larousse.

Celotti, Nadine (2008). «Par des dictionnaires. Droit de cité aux mots des cités». Études de linguistique appliquée, 150, p. 207-220.

Charaudeau, Patrick et Dominique Maingueneau (2002). Dictionnaire d'analyse du discours. Paris, Seuil.

Drouin, Anne Marie (1987). «Des images et des sciences». ASTER, 4, p. 1-32.

Eco, Umberto (1984). La structure absente. Introduction à la recherche sémiotique. Paris, Mercure de France.

Eco, Umberto (1988). Le signe. Bruxelles, Labor.

Eco, Umberto (2007). Dire presque la même chose. Expériences de traduction. Paris, Grasset.

Fabbri, Paolo (2008). Le tournant sémiotique. Paris, Lavoisier.

Gambier, Yves (2016). "Traduction et texte: vers un nouveau double paradigme». Signata, 7, p. 175-197.

Gangla, Lilian-Atieno (2001). Pictorial Illustrations in Dictionaries. MA Thesis. Pretoria, University of Pretoria.

Greimas, Algirdas-Julien (1966). Sémantique structurale. Paris, Larousse.

Greimas, Algirdas Julien (1968). «Conditions d'une sémiotique du monde nature». Langages, 10 , p. 3-35.

Guidère, Mathieu (2008). Introduction à la traductologie. Bruxelles, De Boeck.

Hartmann, Reinhard et Gregory James (1998). Dictionary of Lexicography. Londres et New York, Routledge.

Jakobson, Roman (1963). Essais de linguistique générale. 1. Les fondations du langage. Paris, Minuit.

Joly, Martine (1993). Introduction à l'analyse de l'image. Paris, Nathan.

Ilson, Robert (1987). "Illustrations in Dictionaries». In A. Cowie, dir. The Dictionary and the Language Learner: Papers from the EURALEX Seminar at the University of Leeds, 1-3 April 1985. Tübingen, Niemeyer, p. 193-212.

Klinkenberg, Jean-Marie (1996). Précis de sémiotique générale. Bruxelles, De Boeck Université.

Klosa, Annette (2015). "Illustrations in Dictionaries: Encyclopaedic and Cultural Information in Dictionaries». In P. Durkin, dir. The Oxford Handbook of Lexicography. Oxford, Oxford University Press, p. 515532.

Kourdis, Evangelos et Pirjo Kukkonen (2015). «Introduction. Semiotics of translation, Translation in Semiotics ». Punctum, 1, 2, p. 5-10.

Ladmiral, Jean-René (1994). Traduire : théorèmes pour la traduction. Paris, Gallimard.

Lemonier, Marc (2008). Petit Dico d'argot. Bernay, City Editions.

Lew, Robert (2010). «Multimodal Lexicography: The Representation of 
Meaning in Electronic Dictionaries». Lexikos, 20, p. 290-306.

Margarito, Maria Grazia (2007). «Entre rigueur et agrément : de quelques microstructures de dictionnaires contemporains». In E. Galazzi et C. Molinari, dir. Les français en émergence. Berne, Peter Lang, p. 171182.

Merle, Pierre (1997). L'argus des mots. Paris, L’Archipel.

Munday, Jeremy (2004). «Advertising: Some Challenges to Translation Theory». The Translator, 10, 2, p. 199-219.

Nesi, Hilary (1989). «How Many Words is a Picture Worth? A Review of Illustrations in Dictionaries». In M. L. Tickoo, dir. Learners' Dictionaries: State of the Art. Singapore, SEAMEP Regional Language Center, p. 124-134.

Oittinen, Riitta (2000). Translating for Children. New York et Londres, Garland Publishing Co.

Peirce, Charles Sanders (1979). Écrits sur le signe. Trad. Gérard Deledalle. Paris, Le Seuil.

Pereira, Nilce M. (2008). «Book Illustration as (Intersemiotic) Translation: Pictures Translating Words». Meta, 53, 1, p. 104-119.

Pérez, Marcela (2007). «Avant-propos». Lexik des cités. Paris, Éditions Fleuve Noir, p. 7-9.

Petrilli, Susan et Augusto Ponzio (2012). «Iconicity, Otherness and Translation». Chinese Semiotic Studies, 7, 1, p. 11-26.

Proces, Michel (1997). «L'architecture et la construction: références, structures et apports du dessin». In A. Hermans, dir. Les dictionnaires spécialisés et l'analyse de la valeur. Actes du colloque organisé en avril 1995 par le Centre de terminologie de Bruxelles (Institut Libre Marie Haps). Louvain-la-Neuve, Peeters, p. 145-176.

Rey-Debove,Josette (1971). Étude linguistique et sémiotique des dictionnaires français contemporains. The Hague, Mouton.

Tomaszkiewicz, Teresa (2005). «La traduction intersémiotique fait-elle partie de la traductologie?». In J. Peeters, dir. La traduction. De la théorie à la pratique et retour. Rennes, Presses universitaires de Rennes, p. 159-168.

Valdam, Albert (2000). «La langue des faubourgs et des banlieues: de l'argot au français populaire». The French Revierw, 73, 6, p. 1179-1192.

Wood, Phyllis et Patrick McDonnell (1994). Scientific Illustration: A Guide to Biological, Zoological, and Medical Rendering Techniques, Design, Printing, and Display. New York, John Wiley \& Sons.

Evangelos Kourdis

Département de Langue et Littérature françaises Université Aristote de Thessalonique Thessalonique, Grèce ekourdis@frl.auth.gr 